\title{
Performance of a Cognitive Relay Network under AF Relay Selection Scheme with Imperfect Channel Estimation
}

\author{
Binod PRASAD, Sanjay Dhar ROY, Sumit KUNDU \\ Department of Electronics and Communication, National Institute of Technology, Durgapur, India \\ binod.ece@gmail.com, \{s_dharroy,sumitkundu\}@yahoo.com
}

Manuscript received January 29, 2016

\begin{abstract}
In this paper outage performance of a secondary user (SU) is evaluated under an amplify and forward $(A F)$ relay selection scheme with an imperfect channel state information (CSI) while the SU shares spectrum in an underlay cognitive radio network (CRN). In underlay, the $S U$ coexists with primary user (PU) in the same band provided the interference produced by $S U$ at the $P U$ receiver is below an interference threshold of PU. This limits the transmission power of SU and coverage area. Relays help to improve the performance of $S U$ in such underlay scenario. However relays are also constrained in their transmit power due to interference constraint imposed by $P U$. Closed form expression of the outage probability of $S U$ with maximum transmit power constraint of relay under imperfect CSI is derived. A scaling factor based power control is used for the SU transmitter and the relay in order to maintain the interference constraint at the PU receiver due to imperfect CSI. The impact of several parameters viz. correlation coefficient, channel estimation error, tolerable interference threshold, number of relays and the maximum transmit power constraint of relay on $S U$ performance is investigated. A MATLAB based test bed has also been developed to carry out simulation in order to validate the theoretical results.
\end{abstract}

\section{Keywords}

Cognitive radio, amplify and forward relay, imperfect CSI

\section{Introduction}

Due to rapid growth in the field of wireless communication, the demand for the limited electromagnetic radio spectrum is increasing exponentially, which is causing spectrum scarcity. It has also been reported by FCC that the utilization of electromagnetic radio spectrum allocated to a licensed user i.e. PU is very low [1-3]. Cognitive radio has been proposed as a promising solution for efficient utilization of the underutilized radio spectrum. The cognitive user, also known as SU shares the existing spectrum in two ways i.e. spectrum underlay and spectrum overlay. In underlay mode, the SUs are allowed to access the licensed spectrum allocated to the primary user (PU) as long as the interference produced at the PU receiver due to SU transmission remains below a predefined interference threshold [4]. Due to this stringent transmit power requirement, coverage area of cognitive radio network is limited. Relays are generally used to enhance the system performance. A combination of relay and cognitive radio network is used to enhance the performance of SU in cognitive relay network [5-8]. Amplify and forward relay (AF) is one of the popular cooperative protocols where a relay amplifies the received signal from the source and transmits it to the destination. Outage performance is one of the important performance metrics used to evaluate the performance of SU user. The performance of SU is evaluated under bounded SNR regime i.e. end to end SNR of AF relay which is approximated as the minimum SNR of the two hops i.e. SU to relay and relay to destination [9]. In [10], outage performance of cognitive relay network is evaluated for a given interference constraint of PU in an underlay spectrum sharing mode. In [11], outage performance of cognitive relay is evaluated under hybrid spectrum access (underlay and interweave) approach. Further a closed form expression for the outage probability is also derived in [11]. Outage probability of SU is evaluated under optimal relay selection scheme utilizing quotient of signal to noise ratio of the relay link and the interference generated at PU receiver due to relay in [12]. Further closed form expressions for the outage and bit error probabilities are also derived in [12]. In [13], outage probability of cognitive cooperative relay network using two decode-and-forward relay schemes under the joint outage constraint of primary user and peak transmit power of secondary user is evaluated. Performance of SU in an underlay cognitive relay network under proactive decode and forward relaying scheme has been evaluated under joint constraints of average and peak interference constraint considering Rayleigh faded channel [14]. Performance of SU under AF relay 
selection scheme has been investigated in [15], [16] under the assumption of perfect CSI. In [17], the performance of SU in terms of outage and symbol error rate (SER) has been evaluated for a Rayleigh faded channel considering maximum transmit power constraint of the relay. In the above references, $\mathrm{SU}$ is assumed to perfectly know the CSI of link between SU transmitter (SU-Tx) and PU receiver (PU-Rx).The CSI is used in deriving the transmit power of SU. Similar assumption has been made i.e. assumption of a perfect CSI, between relay and PU receiver (PU-Rx).

However in a practical communication system obtaining the CSI between SU-Tx and PU-Rx is very difficult. Therefore the CSI information available at SU-Tx regarding the link between SU-Tx and PU-Rx is frequently imperfect. In literature there are two ways to model this imperfection in the channel: a correlation based model, considering the impact of feedback delay to reflect the imperfection in the channel [18] and a minimum mean square error estimation where imperfection in the channel is due to channel estimation error [19]. In an underlay mode SU estimates its transmit power based on CSI between SU-Tx and PU-Rx. Therefore PU is expected to suffer an extra interference if SU allocates its transmit power based on imperfect CSI. Suraweera et al. evaluated the mean capacity of SU in underlay scenario based on imperfect CSI due to feedback delay [18]. However in [19] Musavian et al. evaluated the ergodic and outage capacity of SU under peak and average interference constraint assuming SU has channel estimation error of the CSI between SU-Tx and PU-Rx. In [20], impact of imperfect $\mathrm{CSI}$ of the interfering link on PU for partial AF (fixed gain) scheme has been investigated. Further a closed form expression for the outage probability of SU under a given interference probability of PU (i.e. probability that the interference from SU to PU receiver is higher than PU interference threshold) has also been derived. In [21], outage performance of a cognitive relay network with imperfect channel estimation in spectrum sharing model is evaluated. In [22], we evaluated the outage probability of SU for Rayleigh faded channel with proactive decode and forward (DF) relay scheme considering imperfect CSI due to feedback delay and PU interference. The performance of $\mathrm{SU}$ in terms of outage and error probability under imperfect CSI has been investigated with AF relay [23].

In [18-23], the outdated property of the channel is expressed either by a correlation based model or an MMSE based model. In practice, CSI available at SU transmitter contains both the channel estimation error as well as error due to feedback delay. In [24], the capacity of SU is evaluated under both the average and peak interference constraints considering channel estimation error and feedback delay of the channel considering a Rayleigh faded channel. In [17], the performance of SU is evaluated under the maximum transmit power constraint of the relay with perfect CSI. In this paper we extend the analysis of [17] with imperfect CSI model of [24] in presence of multiple AF relays. A power allocation scheme has also been designed to avoid the extra interference on PU caused due to imperfect CSI. In this paper we analyze the outage performance of SU considering an imperfect channel model similar to [24] under AF relay scheme for a given outage constraint of PU. We assume that the maximum allowed transmit power of SU is sufficiently high, i.e. SU acts as a base station. However the relay transmit power may be limited by its maximum transmit power limit. Here we consider a maximum transmit power constraint of the relay while evaluating the outage probability of SU. A power control law in line with [22] is designed for the SU and the secondary relay (SR) in order to protect the primary receiver from the SU and SR transmission due to imperfect CSI. We derived a closed form expression for the outage probability of SU for the given relay scheme under imperfect CSI. More precisely the contributions of our paper are

- Analysis of AF based SR capturing both the effect of channel estimation error and feedback delay associated with imperfect CSI. Such analysis is not available in the literature to the best of our knowledge.

- We derived novel closed form analytical expression for the outage probability of SU under the joint impacts of channel estimation error $\left(\sigma_{e}^{2}\right)$ and feedback delay $(\rho)$ for a given outage constraint of PU.

- The impact of correlation coefficient $(\rho)$, channel estimation error $\left(\sigma_{e}^{2}\right)$ and maximum transmit power of the relay on a scaling factor based power control used at relay and $\mathrm{SU}$ is also indicated.

- The impact of tolerable interference threshold $\left(I_{\mathrm{th}}\right)$, correlation coefficient $(\rho)$, PU outage constraint $(\delta)$ on the SU outage performance is also indicated.

- A MATLAB simulation testbed is developed in order to validate the analytical result.

The remainder of the paper is organized as follow: Section 2 describes the system model considered in this paper. In Sec. 3, necessary analytical formulation is presented and analytical expression for the outage probability of SU is derived. Section 4 shows the numerical results depicting the impact of several parameters on outage probability following our derivation. Finally, Section 5 concludes the paper.

\section{System Model}

The system model considered in this paper consists of a secondary transmitter (SU-Tx), $N_{r}$ number of SR-s, a SU receiver (SU-Rx) and a primary receiver (PU-Rx) as shown in Fig. 1. All the channels are assumed to be independent and identically distributed (i.i.d) Rayleigh faded channel with zero mean and variance one. Our model can be extended for non i.i.d faded channel. However for ease of mathematical tractability we consider an i.i.d faded channel in the present case. Let $h_{s p}, h_{r_{i} p}, h_{s r_{i}}$, and $h_{r_{i} d}\left(i=1,2, . ., N_{r}\right)$ 


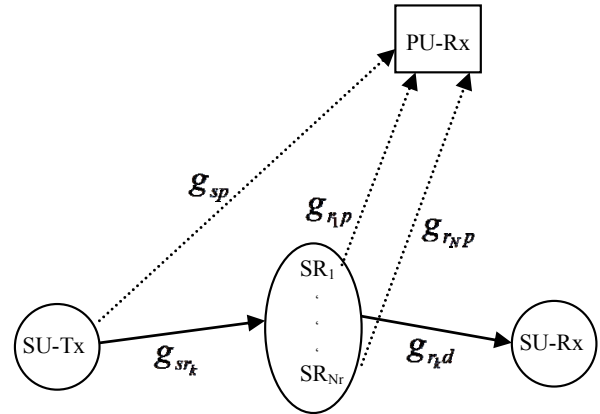

Fig. 1. System model for cognitive relay network.

denote the instantaneous fading coefficients of the links from SU-Tx to PU-Rx, from $i$-th SR to PU-Rx, SU-Tx to $i$ th SR, and $i$-th SR to SU-Rx respectively. Therefore the channel power gain, $g_{\mathrm{xy}}=\left|h_{\mathrm{xy}}\right|^{2}$ is exponentially distributed, where $\mathrm{x}, \mathrm{y}=\{\mathrm{s}, \mathrm{r}\}$ or $\{\mathrm{p}, \mathrm{d}\}$.

We assume that there is no direct link between SU-Tx and SU-Rx. Further it is assumed that SU-Tx and SR do not transmit simultaneously. The communication in the secondary network starts as follows: In the first hop, the relay having the highest SNR among the links between SUTx and SR is selected. The selected SR amplifies the received signal with gain $G$ as in [16] and forwards it to the SU-Rx. The interference from the PU transmitter at the SR and SU-Rx is neglected.

The CSI available at the transmitter of SU and SR are used to estimate the transmit power of SU and SR under a peak interference constraint. However in practical environment, the obtained CSI at the SU $\left(h_{s p}\right)$ and the selected relay $\left(h_{r_{k} p}\right)$ are imperfect due to channel estimation error or feedback delay or due to presence of the both. Therefore, if the transmit power of SU and SR are allocated based on the imperfect CSI, the interference received at the PU receiver may exceed the allowable interference threshold of PU. We consider the imperfect channel model similar to [24] to incorporate the joint impact of channel estimation error $\left(\sigma_{e}^{2}\right.$ and $\sigma^{2}$ ) and correlation coefficient $(\rho)$ as

$$
h_{x y}=\rho \tilde{h}_{x y}+\varepsilon
$$

where $\tilde{h}_{x y} \sim C N\left(0,\left(1-\sigma^{2}\right) \lambda_{x y}\right)$ is an outdated channel impulse response with estimation error, $\varepsilon=\rho \varepsilon_{2}+\varepsilon_{1} \sqrt{1-\rho^{2}} . \quad$ Here $\quad \varepsilon_{1} \sim C N\left(0, \lambda_{\mathrm{xy}}\right) \quad$ and $\varepsilon_{2} \sim C N\left(0, \sigma^{2} \lambda_{\mathrm{xy}}\right)$ are uncorrelated and $\varepsilon \sim C N\left(0, \alpha \lambda_{\mathrm{xy}}\right)$, $\alpha=1-\left(1-\sigma^{2}\right) \rho^{2}$. It is to be noted that $\sigma^{2}\left(0 \leq \sigma^{2} \leq 1\right)$ is a measure of accuracy in the channel estimation from the link $\mathrm{x}$ to $\mathrm{y}$ and $\rho(0 \leq \rho \leq 1)$ is the channel correlation coefficient which accounts for the error due to feedback delay caused by the time varying property of the channel. A lower value of $\rho$ corresponds to a higher feedback delay. Thus following the imperfect channel model as in (1) the CSI available at the SU and SR are given as

$$
h_{s p}=\rho \tilde{h}_{s p}+\varepsilon,
$$

$$
h_{r_{i} p}=\rho \tilde{h}_{r_{i} p}+\varepsilon_{i}
$$

where $\tilde{h}_{s p}$ and $\tilde{h}_{r_{i} p}$ are the imperfect CSI available at the transmitter of SU and $\mathrm{SR}_{\mathrm{i}}$ with $\tilde{h}_{s p} \sim C N\left(0,\left(1-\sigma^{2}\right) \lambda_{s p}\right)$ and $\tilde{h}_{r_{i} p} \sim C N\left(0,\left(1-\sigma_{e}^{2}\right) \lambda_{r_{i} p}\right) \quad$ respectively. Further $\varepsilon$ is $C N\left(0, \alpha \lambda_{s p}\right), \quad \sigma^{2}\left(0 \leq \sigma^{2} \leq 1\right)$ and $\sigma_{e}{ }^{2}\left(0 \leq \sigma_{e}{ }^{2} \leq 1\right)$ are the channel estimation error (i.e. error variance associated with MMSE based channel estimation) for the link from SU-Tx to PU-Rx and the $i$-th relay to PU-Rx respectively. The CSI of the source to relay and relay to destination link are assumed to be perfect. Further we also assume that SU as well as the selected $k$-th relay knows $\tilde{h}_{s p}, \tilde{h}_{r_{i} p}, \sigma_{e}{ }^{2}$ and $\rho$.

Therefore based on the estimate of $h_{s p}$, and $h_{r_{i} p}$, we allocate scaling factor based power to the SU transmitter and $i$-th relay $\left(p_{r_{i}}\right)$ under imperfect CSI following [22]:

$$
p_{s}=\frac{\eta I_{\mathrm{th}}}{\tilde{g}_{s p}} \text { and } P_{r_{i}}=\min \left(\frac{\eta_{r} I_{\mathrm{th}}}{\tilde{g}_{r_{i} p}}, p_{m}\right), i=1,2, \ldots N_{r}
$$

where $\eta(0<\eta \leq 1)$ and $\eta_{r}\left(0<\eta_{r} \leq 1\right)$ are scaling parameters which take care of the excessive interference produced at PU receiver due to $\mathrm{SU}$ and relay transmission under imperfect CSI.

The communication in the secondary network starts as follows. In the first time slot, the SU broadcasts its message to $N_{r}$ SR-s and the relay having the maximum signal to noise ratio of the SU-Tx to SR link is selected. The SNR at the selected $k$-th relay is given as

$$
\gamma_{s r_{k}}=\frac{p_{s} g_{s r_{k}}}{N_{0}}=\frac{\eta I_{\mathrm{th}} g_{s r_{k}}}{\tilde{g}_{s p} N_{0}}
$$

The value of $\eta$ is obtained as per the outage constraint of PU following [22]

$$
\begin{aligned}
P_{\text {out-su }}^{\mathrm{pu}} & =\operatorname{Pr}\left\{p_{s} g_{s p}>I_{\mathrm{th}}\right\} \leq \delta \\
& =\operatorname{Pr}\left\{\frac{g_{s p}}{\tilde{g}_{s p}} \geq \frac{1}{\eta}\right\}
\end{aligned}
$$

where $\delta$ is the allowable outage constraint of PU and $P_{\text {out-su }}^{\text {pu }}$ indicates the outage probability of PU due to SU transmission.

Now using the joint pdf of $g_{s p}$ and $\tilde{g}_{s p}$ as given in [25]

$$
f_{g_{s p} \tilde{g}_{s p}}\left(g_{s p}, \tilde{g}_{s p}\right)=\frac{1}{\left(1-\sigma^{2}\right) \alpha \lambda_{s p}^{2}} \mathrm{e}^{\left(-\frac{\left(1-\sigma^{2}\right) g_{s p}+\tilde{g}_{s p}}{\left(1-\sigma^{2}\right) \alpha \lambda_{s p}}\right)} I_{0}\left(\frac{2 \rho \sqrt{g_{s p} \tilde{g}_{s p}}}{\alpha \lambda_{s p}}\right)
$$

where $I_{0}($.$) is the zeroth-order modified Bessel function of$ first kind [26], equation (6) can be solved for $\eta$ as

$$
\eta=\left(1-\sigma^{2}\right)\left(1+\frac{2 \alpha \theta}{1-\theta}+\sqrt{\left(\frac{2 \alpha \theta}{1-\theta}+1\right)^{2}-1}\right)
$$


where $\theta=(1-2 \delta)^{2}$.

In the second time slot, the selected $k$-th relay amplifies the received signal and forwards it to the SU destination. The SNR at the SU destination is given as

$$
\gamma_{r_{k} d}=\frac{p_{r_{k}} g_{r_{k} d}}{N_{0}}
$$

where $p_{r_{k}}=\min \left(\frac{\eta_{r_{k}} I_{\text {th }}}{\tilde{g}_{r_{k} p}}, p_{m}\right), k=1,2, \ldots N_{r}$,

Further the value of $\eta_{r_{k}}$ is obtained for the given outage constraint $(\delta)$ of PU as follows

$$
\begin{aligned}
P_{\text {outrel }}^{\text {pu }} & =\operatorname{Pr}\left\{p_{r_{k}} g_{r_{k} p}>I_{\text {th }}\right\} \leq \delta \\
& =\operatorname{Pr}\left\{\frac{\eta_{r} I_{\text {th }}}{\tilde{g}_{r_{k} p}} g_{r_{k} p}>I_{\text {th }}\right\} \leq \delta \\
& =\int_{\frac{I_{t h}}{P_{m}}}^{\infty} \int_{0}^{\eta x} f_{g_{k_{p}} \tilde{s}_{r_{k} p}}(x, y) \mathrm{d} y \mathrm{~d} x \leq \delta
\end{aligned}
$$

where $P_{\text {out-rel }}$ is is the outage probability of PU due to relay transmission and $f_{x}($.$) denotes the probability density$ function of $\mathrm{x}$. Now using the joint pdf of $g_{r_{k} p}$ and $\hat{g}_{r_{k} p}$ as in

$$
f_{g_{k p p} \tilde{g}_{k p p}}\left(g_{r_{k} p}, \tilde{g}_{r_{k} p}\right)=\frac{1}{\left(1-\sigma_{e}^{2}\right) \alpha \lambda_{s p}{ }^{2}} \mathrm{e}^{\left(-\frac{\left(1-\sigma_{e}^{2}\right) g_{k p}+\tilde{g}_{k p}}{\left(1-\sigma_{e}^{c}\right) \alpha \lambda_{p p}}\right)} I_{0}\left(\frac{2 \rho \sqrt{g_{r_{k} p} \tilde{g}_{r_{k} p}}}{\alpha \lambda_{s p}}\right)
$$

where $I_{0}($.$) is the zeroth-order modified Bessel function of$ first kind [26], equation (9) can be simplified as

$$
\begin{aligned}
P_{\text {out-rel }}^{\text {pu }} & =e^{-\frac{I_{\mathrm{th}}}{p_{m} \lambda_{k p}}}-e^{-\frac{p c^{2}}{2}} Q(a c, b c)+ \\
& \frac{t}{r} Q\left(c \sqrt{\frac{s-r}{2}}, c \sqrt{\frac{s+r}{2}}\right)-\frac{1}{2}\left(1+\frac{t}{r}\right) e^{-\frac{s c^{2}}{2}} I_{0}\left(a b c^{2}\right)
\end{aligned}
$$

where

$$
\begin{aligned}
& a=\sqrt{\frac{2 \rho^{2}\left(1-\sigma_{e}^{2}\right)}{\alpha \lambda_{r_{k} p}}}, b=\sqrt{\frac{2 \eta_{r}}{\left(1-\sigma_{e}^{2}\right) \alpha \lambda_{r_{k} p}}}, c=\sqrt{\frac{I_{\text {th }}}{p_{m}}}, \\
& s=\frac{2}{\lambda_{r_{k} p}}\left(1+\frac{\rho^{2}\left(1-\sigma_{e}^{2}\right)}{\alpha}+\frac{\eta_{r}}{\left(1-\sigma_{e}^{2}\right) \alpha}\right), \\
& t=\frac{2}{\lambda_{r_{k} p}}\left(1+\frac{\rho^{2}\left(1-\sigma_{e}^{2}\right)}{\alpha}-\frac{\eta_{r}}{\left(1-\sigma_{e}^{2}\right) \alpha}\right)
\end{aligned}
$$

and

$$
\begin{aligned}
r & =\frac{2}{\alpha \lambda_{r_{k} p}\left(1-\sigma_{e}^{2}\right)} \\
& \sqrt{\left(\alpha\left(1-\sigma_{e}^{2}\right)+\rho^{2}\left(1-\sigma_{e}^{2}\right)^{2}+\eta_{r}\right)^{2}-4 \rho^{2} \eta_{r}\left(1-\sigma_{e}^{2}\right)^{2}}
\end{aligned}
$$

It is difficult to obtain a closed form expression for the scaling factor $\left(\eta_{r}\right)$ for a given outage constraint $(\delta)$ of PU. So we evaluate (10) numerically to obtain $\eta$ for a given $\delta$.

The SNR of the source relay destination link under AF relay can be written as [27]

$$
\gamma_{\mathrm{end}}^{\mathrm{AF}}=\frac{\gamma_{s r_{k}} \gamma_{r_{k} d}}{1+\gamma_{s r_{k}}+\gamma_{r_{k} d}}
$$

\section{Outage Probability Analysis}

The outage probability of SU is defined as the probability that the mutual information between the transmitted message and the received message is less than a given target rate, $R$ [28]. The outage probability of SU under $\mathrm{AF}$ relaying is given as

$$
\begin{aligned}
& P_{\text {out-su }} \mathrm{AF}=\operatorname{Pr}\left\{\frac{\gamma_{s_{k}} \gamma_{r_{k} d}}{1+\gamma_{s_{k}}+\gamma_{r_{k} d}} \leq \gamma_{\mathrm{th}} \cap \max _{r_{i} \in N, i \neq k} g_{s_{i}}<g_{s_{r_{k}}}\right\} \\
& =\sum_{k=1}^{N} \operatorname{Pr}\left\{\frac{\gamma_{s_{k}} \gamma_{r_{k} d}}{1+\gamma_{s_{k}}+\gamma_{r_{k} d}} \leq \gamma_{\mathrm{th}} \cap \max _{r_{i} \in N, i \neq k} g_{s r_{i}}<g_{s_{k}}\right\} \\
& =N \int_{0}^{\infty} \operatorname{Pr}\left\{\frac{\gamma_{s_{k}} u}{1+\gamma_{s_{k}}+u} \leq \gamma_{\mathrm{th}}\right\} \operatorname{Pr}\left\{\max _{r_{i} \in N, i \neq k} g_{s_{i}}<z\right\} f_{g_{s_{k}}}(z) \\
& =N \int_{0}^{\infty} \underbrace{\operatorname{Pr}\left\{\frac{\gamma_{s_{k}} u}{1+\gamma_{s_{k}}+u} \leq \gamma_{\mathrm{th}}\right\}}_{I_{1}} \underbrace{\operatorname{Pr}\left\{\max _{r_{i} \in N, i \neq k} g_{s_{i}}<z\right\}}_{I_{2}} f_{g_{s_{k}}}(z)
\end{aligned}
$$

Now $I_{1}$ and $I_{2}$ of the above equation (12) are given as

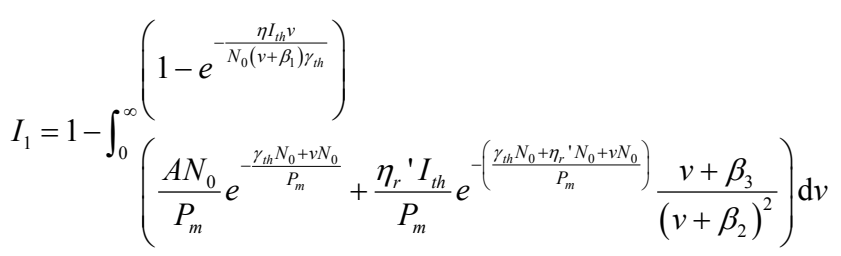

where

$$
\begin{gathered}
f_{\gamma_{k d}}(u)=\left(A N_{0} / P_{m}\right) e^{-\frac{u N_{0}}{P_{m}}}+ \\
\frac{N_{0} \eta_{r}{ }^{\prime} I}{P_{m}} e^{-\left(\frac{\eta^{\prime} r^{\prime} I u N_{0}}{P_{m}}\right)} \frac{u N_{0}+P_{m}+\eta_{r}{ }^{\prime} I}{\left(u N_{0}+\eta_{r}{ }^{\prime} I\right)^{2}} \\
A=1-\exp \left(-\frac{\eta_{r}{ }^{\prime} I}{p_{m}}\right), \eta_{r}{ }^{\prime}=\frac{\eta_{r}}{1-\sigma_{e}{ }^{2}}, \\
I_{2}=\operatorname{Pr}\left\{\max _{r_{i} \in N, i \neq k} g_{s r_{i}}<z\right\}=\sum_{j=0}^{N-1}\left(\begin{array}{c}
N \\
j
\end{array}\right)(-1)^{j} \exp (-j z) .
\end{gathered}
$$

Now substituting $I_{1}$ and $I_{2}$ in (12), the outage probability of $\mathrm{SU}$ is given as

$$
P_{\text {out }}=\sum_{j=0}^{N-1}\left(\begin{array}{c}
N-1 \\
j
\end{array}\right)(-1)^{j} \frac{1}{j+1}-\left(I_{3}+I_{4}-I_{5}-I_{6}\right)
$$

where $I_{3}, I_{4}, I_{5}$ and $I_{6}$ are given below as 


$$
\begin{aligned}
& I_{3}=A \exp \left(-\frac{\gamma_{\mathrm{th}} N_{0}}{P_{m}}\right) \sum_{j=0}^{N-1}\left(\begin{array}{c}
N-1 \\
j
\end{array}\right)(-1)^{j} \frac{1}{j+1}, \\
& I_{4}=\frac{\eta_{r}{ }^{\prime} I_{\text {th }}}{P_{m}} e^{-\left(\frac{\eta_{r}{ }^{\prime} I_{\text {th }}}{P_{m}}+\frac{\gamma_{\mathrm{th}} N_{0}}{P_{m}}\right)} \sum_{j=0}^{N-1}\left(\begin{array}{c}
N-1 \\
j
\end{array}\right)(-1)^{j} \frac{1}{j+1} \\
& \times\left(e^{\frac{\beta_{2} N_{0}}{P_{m}}} E i\left(-\frac{\beta_{2} N_{0}}{P_{m}}\right)\left(\left(\beta_{3}-\beta_{2}\right) \frac{N_{0}}{P_{m}}-1\right)+\frac{\left(\beta_{3}-\beta_{2}\right)}{\beta_{2}}\right) \\
& I_{5}=\frac{\gamma_{\mathrm{th}} A N_{0}^{2} e^{-\frac{\gamma_{\mathrm{th}} N_{0}}{P_{m}}}}{P_{m}\left(N_{0} \gamma_{\mathrm{th}}(j+1)+\eta^{\prime} I_{\mathrm{th}}\right)} \\
& \times\left(\beta_{4} e^{\frac{\beta_{4} N_{0}}{P_{m}}} E i\left(-\frac{\beta_{4} N_{0}}{P_{m}}\right)+\frac{P_{m}}{N_{0}}-\beta_{1} e^{\frac{\beta_{4} N_{0}}{P_{m}}} E i\left(-\frac{\beta_{4} N_{0}}{P_{m}}\right)\right)
\end{aligned}
$$

where $\eta^{\prime}=\frac{\eta}{1-\sigma^{2}}$.

$$
\begin{aligned}
& I_{6}=\eta I_{\mathrm{th}} \gamma_{\mathrm{th}} N_{0} e^{-\left(\frac{\eta_{r} I_{\mathrm{th}}+\gamma_{\mathrm{th}} N_{0}}{P_{m}}\right)} \\
& \left(e^{\frac{\beta_{2} N_{0}}{P_{m}}} E i\left(-\frac{\beta_{2} N_{0}}{P_{m}}\right)\left(\frac{P N_{0}}{P_{m}}-M\right)-L e^{\frac{\beta_{5} N_{0}}{P_{m}}} E i\left(-\frac{\beta_{5} N_{0}}{P_{m}}\right)\right) \\
& \sum_{j=0}^{N-1}\left(\begin{array}{c}
N-1 \\
j
\end{array}\right)(-1)^{j} \frac{1}{P_{m}\left(\gamma_{\text {th }}(j+1)+\eta^{\prime} I_{\text {th }}\right)}
\end{aligned}
$$

and

$\beta_{1}=\gamma_{\text {th }}+1, \beta_{2}=\frac{\gamma_{\text {th }} N_{0}+\eta_{r}^{\prime} I_{\text {th }}}{N_{0}}, \beta_{3}=\frac{P_{m}+\eta_{r}^{\prime} I_{\text {th }}+N_{0} \gamma_{\text {th }}}{N_{0}}$,

$\beta_{4}=\frac{\gamma_{\mathrm{th}} \beta_{1}(j+1) N_{0}}{N_{0} \gamma_{\mathrm{th}}(j+1)+\eta^{\prime} I_{\mathrm{th}}}, \beta_{5}=\frac{\gamma_{\mathrm{th}} \beta_{1}(j+1)}{\gamma_{\mathrm{th}}(j+1)+\eta^{\prime} I_{\mathrm{th}}}$,

$L=\frac{\left(\beta_{3}-\beta_{5}\right)\left(\beta_{1}-\beta_{5}\right)}{\left(\beta_{2}-\beta_{5}\right)^{2}}$,

$M=\frac{\left(\beta_{3}-\beta_{2}\right)\left(\beta_{5}-\beta_{1}\right)+\left(\beta_{5}-\beta_{2}\right)\left(\beta_{1}-\beta_{2}\right)}{\left(\beta_{5}-\beta_{2}\right)^{2}}$,

$P=\frac{\left(\beta_{3}-\beta_{2}\right)\left(\beta_{1}-\beta_{2}\right)}{\left(\beta_{5}-\beta_{2}\right)}$.

Thus equation (14) represents a novel closed form expression for the outage probability of SU as developed by us.

\section{Numerical Results}

In this section the numerical results in terms of closed form expression of the outage probability of SU as derived in Sec. 3 are validated by MATLAB simulation. The simulation parameters considered are as follows: SU target rate, $R=1 \mathrm{bps} / \mathrm{Hz}, \lambda_{s p}=\lambda_{s r_{i}}=\lambda_{r_{i} d}=\lambda_{r_{i} p}=1, N_{0}=-20 \mathrm{~dB}$.

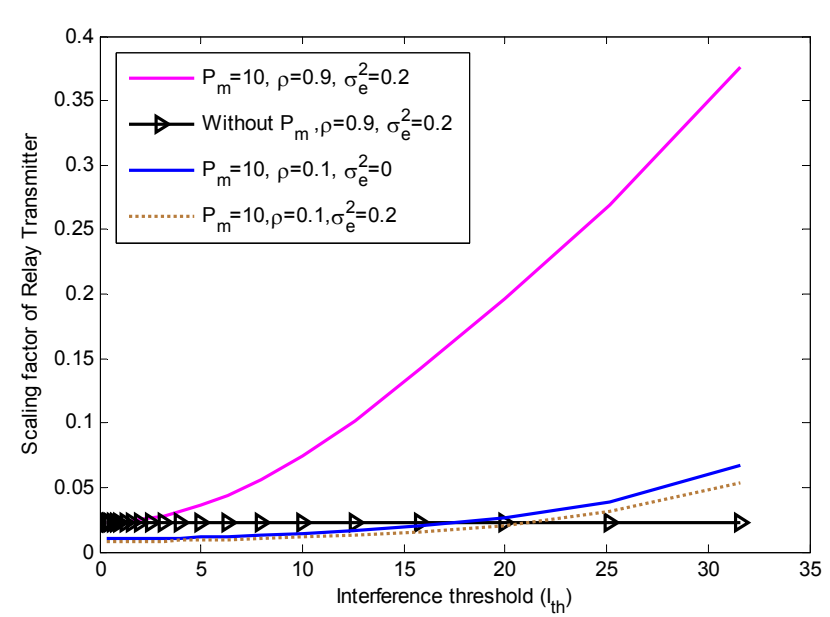

Fig. 2. Scaling factor of relay versus allowable interference threshold $(\delta=0.01)$

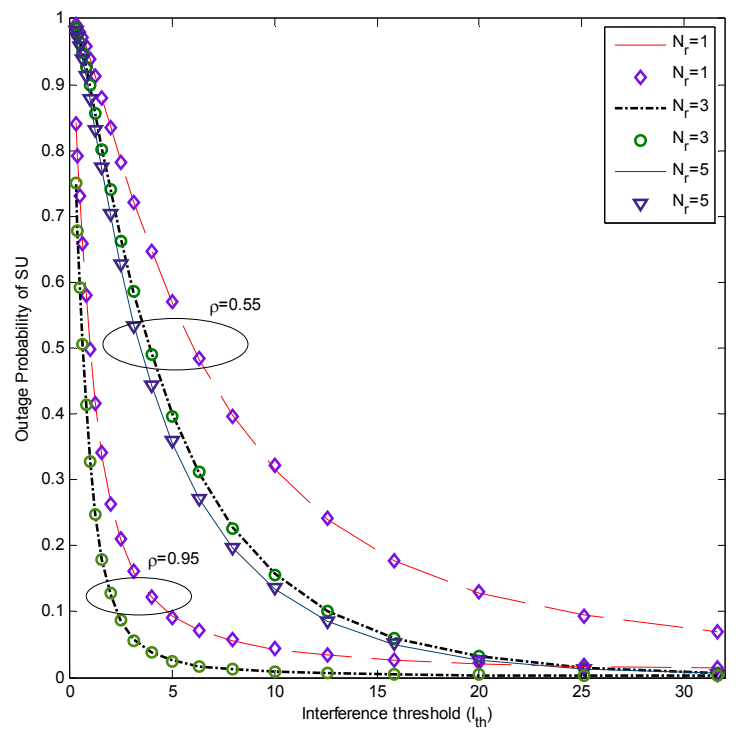

Fig. 3. Outage probability of SU versus allowable interference threshold $\left(\delta=0.01, \sigma^{2}=0, \sigma_{e}^{2}=0, P_{m}=10 \mathrm{~dB}\right)$.

In Fig. 2 the scaling factor $\left(\eta_{r}\right)$ to be used in power control of the relay transmitter is plotted against the allowable interference threshold for different levels of maximum transmit power $\left(P_{m}\right)$ of relay. It is observed that the value of scaling factor of the relay increases as the allowable interference threshold $\left(I_{\mathrm{th}}\right)$ increases. However the value of scaling factor is more in the case where the relay has a maximum transmit power constraint as compared to the case of without maximum transmit power constraint. Further it is observed that the required scaling factor for power control reduces as the maximum transmit power of the relay $\left(P_{m}\right)$ increases since the probability of transmitting with $P_{m}$ decreases. Further the impact of channel correlation coefficient $(\rho)$ and estimation error $\left(\sigma_{e}^{2}\right)$ on scaling factor of the relay is also indicated. As the channel correlation coefficient $(\rho)$ increases, the scaling factor of relay increases. Higher value of $\rho$ indicates the estimation is approaching towards more perfect information of the channel state (i.e. a better CSI) while lower value of $\sigma_{e}{ }^{2}$ indicates a better CSI or reduction in imperfection of CSI estimation. 
The joint impact of number of relays $\left(N_{r}\right)$ and channel correlation coefficient $(\rho)$ on SU outage is shown in Fig. 3 The outage probability of SU against allowable interference threshold is plotted in Fig. 3 for several values of $N_{r}$ and $\rho$. The outage probability of SU improves as the number of relays $\left(N_{r}\right)$ increases. It is also observed that higher value of $\rho$ yields an improvement in SU performance. An increase in correlation coefficient indicates a better estimation of CSI which implies that SU can transmit at higher power level without violating the interference constraint $\left(I_{\text {th }}\right)$ imposed on PU. The outage probability of SU is also plotted for the perfect CSI case and it is compared with imperfect CSI case for different levels of channel imperfections.

The performance of SU for several values of channel estimation error of the source and the relay link $\left(\sigma^{2}\right.$ and $\sigma_{e}^{2}$ ) while keeping other parameters fixed is plotted in Fig. 4. As the channel estimation error increases $\left(\sigma^{2}\right.$ or $\left.\sigma_{e}^{2}\right)$ the outage probability of SU increases. An increase in $\sigma^{2}$ or $\sigma_{e}^{2}$ due to increase in channel estimation error yields a reduction in the transmit power of $\mathrm{SU}$ or the selected relay and this in turn results in an increase in the outage probability of SU. The performance of SU is better when MMSE based estimation error is zero, i.e. the channel is perfectly estimated though a correlation error due to feedback delay $(\rho=0.9)$ is present. It is also observed that the performance of SU is better for the same level of estimation error (i.e. $\sigma^{2}=0.3, \sigma_{e}^{2}=0$ ) in the $\mathrm{SU}$ to PU link as compared to the estimation error (i.e. $\sigma^{2}=0, \sigma_{e}^{2}=0.3$ ) in the relay to PU link.

The impact of PU outage constraint $(\delta)$ on SU outage probability is shown in Fig. 5. The performance of SU is better for higher values of $\delta$ as compared to the lower values of $\delta$. As $\delta$ increases the allowable transmit power of SU increases and hence an improvement in the outage probability of SU is obtained.

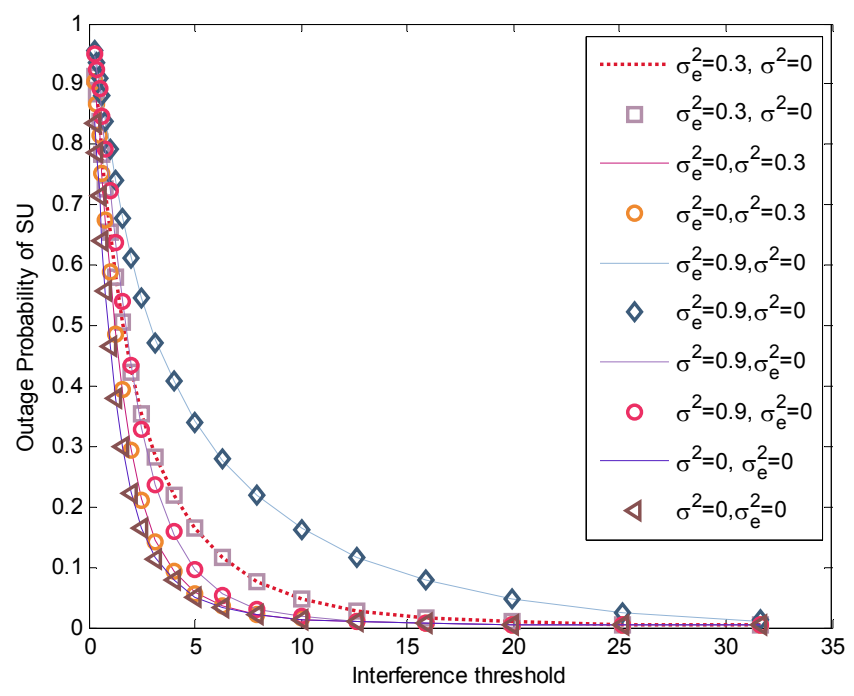

Fig. 4. Outage probability of SU versus allowable interference threshold $\left(\delta=0.01, \rho=0.9, N_{r}=9, P_{m}=10 \mathrm{~dB}\right)$.

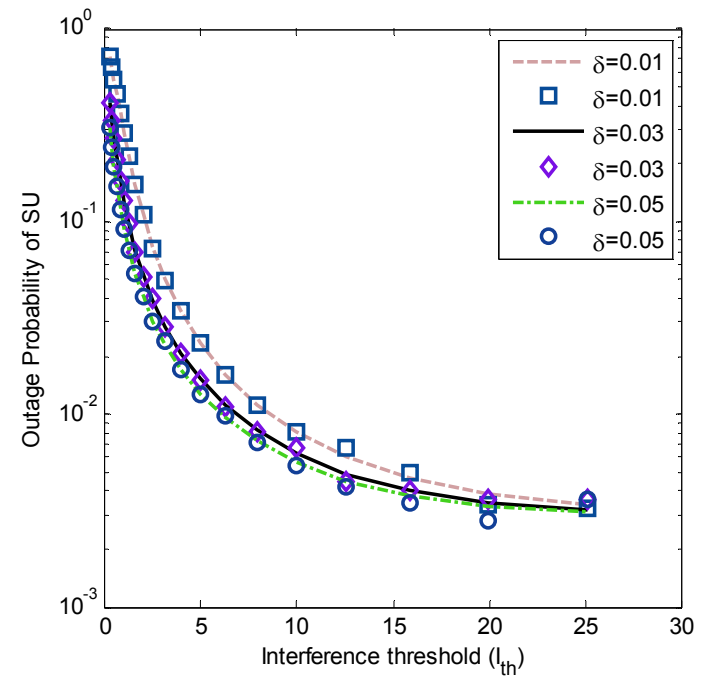

Fig. 5. Outage probability of SU versus allowable interference threshold ( $\left.\rho=0.95, N_{r}=5, P_{m}=10 \mathrm{~dB}, \sigma^{2}=0, \sigma_{e}^{2}=0\right)$.

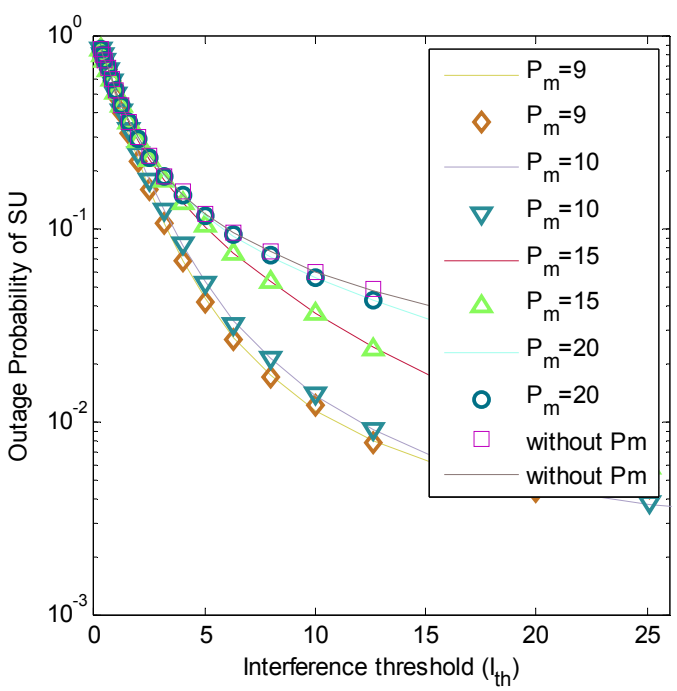

Fig. 6. Outage probability of SU versus allowable interference threshold ( $\rho=0.9, N_{r}=5, \delta=0.01, \sigma^{2}=0, \sigma_{e}^{2}=0$ ).

The impact of maximum transmit power $\left(P_{m}\right)$ of the relay on SU performance is indicated in Fig. 6. It is observed that the performance of SU improves with $P_{m}$ as compared to the case without $P_{m}$. Further it is also observed that the performance of SU degrades as $P_{m}$ increases. As $P_{m}$ increases, the probability of transmission at a level of $P_{m}$ by the relay reduces which increases the outage probability of SU following the explanation given in connection of Fig. 2.

\section{Conclusion}

In this paper performance of a cognitive relay network using an AF based SR is evaluated in terms of outage probability. An AF relay is selected based on the quality of the links between SU-Tx and relays. The link between the selected $k$-th relay to PU-Rx is assumed to be imperfect due to feedback delay as well as channel estimation error. 
We derived an exact closed form expression of the outage probability of SU with AF relay for a given outage constraint of PU. The impact of feedback delay and the channel estimation error on SU outage probability is evaluated. It is observed that the performance of SU degrades as the channel estimation error or feedback delay increases. However the use of relay yields an improvement in the outage probability of SU. The impact of maximum allowed transmit power of relay, allowable interference threshold and PU outage constraint is also indicated. The performance degradation due to imperfect CSI can be compensated by increase in number of available relays $\left(N_{r}\right)$ in the selection set.

\section{References}

[1] HAYKIN, S. Cognitive radio: brain-empowered wireless communications. IEEE Journal on Selected Areas in Communication, 2005, vol. 23, no. 2, p. 201-220. DOI: 10.1109/JSAC.2004.839380

[2] FCC Spectrum Policy Task Force Report, FCC02-155. Nov. 2002.

[3] GHASEMI, A., SOUSA, E. S. Fundamental limits of spectrumsharing in fading environments. IEEE Transactions on Wireless Communication, 2007, vol. 6, no. 2, p. 649-658. DOI: 10.1109/TWC.2007.05447

[4] MOLISCH, A. F., GREENSTEIN, L. J., SHAFI, M. Propagation issues for cognitive radio. Proceedings of the IEEE, 2009, vol. 97 no. 5, p. 787-804. DOI: 10.1109/JPROC.2009.2015704

[5] LANEMAN, J.N., TSE, D.N.C., WORNELL, W. G. Cooperative diversity in wireless networks: Efficient protocols and outage behaviour. IEEE Transactions on Information Theory, 2004, vol. 50, no. 12, p.3062-3080. DOI: 10.1109/TIT.2004.838089

[6] ZHANG, Q., JIA, J., ZHANG, J. Cooperative relay to improve diversity in cognitive radio networks. IEEE Communication Magazine, 2009, vol. 47, no. 2, p. 111-117. DOI: 10.1109/MCOM.2009.4785388

[7] ZHANG, W., BEN LETAIEF, K. Cooperative communications for cognitive radio networks. Proceedings of IEEE, 2009, vol. 97, no. 5, p. 878-893. DOI: 10.1109/JPROC.2009.2015716

[8] RENK, T., JAEKEL, H., JONDRAL, F.K., GOLDSMITH, A., et al. Do decode-and-forward relaying protocols beat transmit diversity?. In 2010 European Wireless Conference (EW). Lucca (Italy), 2010, p. 294-300. DOI: 10.1109/EW.2010.5483434

[9] DING, H., GE, J., DA COSTA, D.B., JIANG, Z. Asymptotic analysis of cooperative diversity systems with relay selection in a spectrum sharing scenario. IEEE Transactions on Vehicular. Technology, 2011, vol. 60, no. 2, p. 457-472. DOI: 10.1109/TVT.2010.2100053

[10] LEE, J., WANG, H., ANDREWS, J., HONG, D., et al. Outage probability of cognitive relay networks with interference constraints. IEEE Transactions on Wireless Communication, 2011, vol. $10, \quad$ no. $2, \quad$ p. $390-395 . \quad$ DOI: 10.1109/TWC.2010.120310.090852

[11] YUAN FU, WANG, Z., XIANG, L.J., ZHENG, L.H., et al. Outage performance of cognitive relay networks using interweaveunderlay approach. In Second International Conference on Digital Manufacturing and Automation (ICDMA). Hunan (China), 2011, p. 833-836. DOI: 10.1109/ICDMA.2011.205

[12] HUSSAIN, S. I., ABDALLAH, M. M., ALOUINI, M. S., HASNA M. O., et al. Best relay selection using SNR and interference quotient for underlay cognitive networks. In Proceedings of the IEEE International Conference on Communications (ICC). Ottawa (Canada), 2012, p. 4176-4180. DOI: 10.1109/ICC.2012.6364463

[13] TRAN, H., ZEPERNICK, H.J., PHAN, H. Cognitive proactive and reactive DF relaying schemes under joint outage and peak transmit power constraints. IEEE Transactions on Wireless Communication, 2011, vol. 17, no. 8, p. 1548-1551. DOI: 10.1109/LCOMM.2013.062113.130573

[14] HUANG, H.Y., SI, J.B., LI, Z., HAO, B.J., et al. Performance analysis of cognitive relay networks under both average and peak interference power constraints. In Proceedings of the IEEE International Conference on Communications in China (ICCC). 2013, p. 297-302. DOI: 10.1109/ICCChina.2013.6671132

[15] DUONG, T.Q., BAO, V.N.Q., ALEXANDROPOULOS, G.C., ZEPERNICK, H.J., et al. Cooperative spectrum sharing networks with AF relay and selection diversity. Electronics Letters, 2011, vol. 47, no. 20, p. 1149-1151. DOI: 10.1049/el.2011.2592

[16] DUONG, T.Q., BAO, V.N.Q., ZEPERNICK, H.-J. Exact outage probability of cognitive AF relaying with underlay spectrum sharing. Electronics Letters, 2011, vol. 47, no. 17, p. 1001-1002. DOI: $10.1049 / \mathrm{el} .2011 .1605$

[17] YU, H., TANG, W., LI, S. Outage probability and SER of amplifyand-forward cognitive relay networks. IEEE Wireless Communication Letter, 2013, vol. 2, no. 2, p. 219-222. DOI: 10.1109/WCL.2013.012513.120834

[18] SURAWEERA, H. A., SMITH, P. J., SHAFI, M. Capacity limits and performance analysis of cognitive radio with imperfect channel knowledge. IEEE Transactions on Vehicular. Technology, 2010, vol. 59, no. 4, p. 1811-1822. DOI: 10.1109/TVT.2010.2043454

[19] MUSAVIAN, L., AISSA, S. Fundamental capacity limits of cognitive radio in fading environments with imperfect channel information. IEEE Transactions on Communications, 2009, vol. 57 no. 11 , p. 3472-3480. DOI: 10.1109/TCOMM.2009.11.070410

[20] CHEN, J., SI, J., LI, Z., HUANG, H. On the performance of spectrum sharing cognitive relay networks with imperfect CSI. IEEE Communications Letters, 2012, vol. 16, no. 7, p. 1002-1005. DOI: 10.1109/LCOMM.2012.042512.120100

[21] ZHANG, X., XING, J., YAN, Z., GAO, Y., et al. Outage performance study of cognitive relay networks with imperfect channel knowledge. IEEE Communications Letters, 2013, vol. 17, no. 1, p. 27-30. DOI: 10.1109/LCOMM.2012.112812.121371

[22] PRASAD, B., ROY, S.D., KUNDU, S. Outage performance of cognitive relay network with imperfect channel estimation under proactive DF relaying. In Proceedings of IEEE National Conference on Communications (NCC). Kanpur, 2014, 6 p. DOI: 10.1109/NCC.2014.6811257

[23] HADZI-VELKOV, Z., MICHALOPOULOS, D. S., KARAGIANNIDIS, G. K., SCHOBER, R. Dual-hop amplify-and-forward transmission with imperfect channel estimates at the relay. In $I E E E$ International Conference on Communications (ICC). Ottawa (Canada), 2012, p. 4110-4115. DOI: 10.1109/ICC.2012.6364439

[24] XU, D., FENG, Z., ZHANG, P. On the impacts of channel estimation errors and feedback delay on the ergodic capacity for spectrum sharing cognitive radio. Wireless Personal Communications, 2013, vol. 72, no. 4, p 1875-1887. DOI: 10.1007/s11277-013-1100-5

[25] SIMON, M. K., ALOUINI, M. S. Digital Communication over Fading Channels. New York: Wiley, 2000. ISBN: 9780471649533

[26] GRADSHTEYN, I., RYZHIK, I. Table of Integrals, Series, and Products. Washington: Academic Press, 2007. ISBN: 0-12$373637-4$ 
[27] ZHAO, Y., ADVE, R., LIM, T. J. Improving amplify-and forward relay networks: optimal power allocation versus selection. IEEE Transactions on Wireless Communication, 2007, vol. 6, no. 8, p. 3114-3123. DOI: 10.1109/TWC.2007.06026

[28] YAN, Z., ZHANG, X., WANG, W. Exact outage performance of cognitive relay networks with maximum transmit power limits. IEEE Communication Letter, 2011, vol. 15, no. 12, p. 1317-1319. DOI: 10.1109/LCOMM.2011.103111.111563

\section{About the Authors ...}

Binod PRASAD was born in 1985 . He received his B. Tech. degree in Electronics and Communication Engineering from Asansol Enginnering College, Kanyapur, West Bengal, India in the year 2006. He received the M. Tech degree from Kalyani Government Engineering College, Kalyani, West Bengal, India in the year 2011. Presently, he is attached with the National Institute of Technology, Durgapur, West Bengal, India, as Institute Ph.D Research Scholar in the Department of Electronics and Communication Engineering. His research interest includes Cognitive Radio Networks with spectrum sharing under different fading channels.

Sanjay Dhar ROY was born in Balurghat, West Bengal, India in 1974. He received his B.E. (Hons.) degree in Electronics and Telecommunication Engineering in 1997 from Jadavpur University, Kolkata, India, M.Tech. degree in Telecommunication Engineering in 2008, and Ph.D. degree in Wireless Communication in 2011 from NIT Durgapur. He worked for Koshika Telecom Ltd. from 1997 to 2000. After that he joined the Department of Electronics and Communication Engineering, National Institute of Technology Durgapur as a Lecturer in 2000 and is currently an Assistant Professor there. His research interests include radio resource management, handoff, smart antenna techniques, beamforming and cognitive radio networks. As of today, he has published 40 research papers in various journals and conferences. Mr. Dhar Roy is a member of IEEE (Communication Society) and is a reviewer of IET Communications, Electronics Letters and Journal of PIER, IJCS, Wiley.

Sumit KUNDU received his B.E. (Hons.) degree in Electronics and Communication Engineering in 1991 from NIT, Durgapur, India, M.Tech. degree in Telecommunication Systems Engineering, and Ph.D. in Wireless Communication Engineering from IIT Kharagpur, India, respectively. He has been a faculty in the Department of ECE, National Institute of Technology, Durgapur since 1995 and is currently a Professor there. His research interests include radio resource management in wireless networks, wireless ad hoc and sensor networks, and cognitive radio networks, energy harvesting and physical layer security. As of today, he has published 140 research papers in various journals and conferences. He is a senior member of IEEE (Communication Society) and is a reviewer of several IEEE journals. 